\title{
The value of transvaginal 4-dimensional hysterosalpingo-contrast sonography in predicting the necessity of assisted reproductive technology for women with tubal factor infertility
}

\author{
Pan $\mathrm{Gu}^{1}$, Xuan Yang ${ }^{2}$, Xingping Zhao ${ }^{1}$, Dabao $\mathrm{Xu}^{1}$ \\ ${ }^{1}$ Department of Gynecology, Third Xiangya Hospital, Central South University, Changsha, China; ${ }^{2}$ Xiangya School of Medicine, Central South \\ University, Changsha, China
}

Correspondence to: Dabao Xu; Xingping Zhao. Department of Gynecology, Third Xiangya Hospital, Central South University, 138 Tongzipo Road, Changsha 410013, China. Email: dabaoxu@yahoo.com; zxp8846@126.com.

Background: More than 50 million women suffer from infertility worldwide, among whom $30 \%$ have associated fallopian tube pathology. Fortunately, the diagnostic accuracy of tubal patency has been enhanced with the consistent development of ultrasound imaging technology, especially the invention of transvaginal 4-dimensional hysterosalpingo-contrast sonography (TV 4D HyCoSy). However, detailed imaging data for evaluating the tubal condition for spontaneous conception and assessing the necessity of assisted reproductive technology (ART) have yet to be amassed.

Methods: Patients with tubal factor infertility (TFI) who received TV 4D HyCoSy were recruited for this study. They were divided into two groups according to the method of conception: the natural pregnancy group (patients who naturally conceived within 3 months after TV 4D HyCoSy) and the assisted reproduction group (patients who failed to conceive naturally within the 3 months but successfully conceived through ART). Logistic regression analysis was performed to examine the data obtained from participants' medical history and TV 4D HyCoSy investigation.

Results: Of the initial 1,433 women, 348 were excluded due to exclusion criteria or lack of follow-up. A total of 1,085 TFI patients were finally included, with individuals in the natural pregnancy group accounting for $27.74 \%(n=301)$, and those in the ART group accounting for $37.33 \%(n=405)$. The age was younger and the duration of infertility was shorter in the group of women who conceive spontaneously after TV 4D HyCoSy $(\mathrm{P}<0.05)$. In terms of imaging data, their endometrial thickness was thinner, right fallopian tube wall was more intact, morphology of the right fallopian tube was smoother, and their ovarian motility (bilateral), fallopian tube visualization (bilateral) and overflow condition of the contrast agent from the fimbriae of fallopian tube (bilateral) were better. In addition, the resistance of the contrast agent injection was less likely to be persistent, reflux was less likely to happen and $0 / 1$ dispersion of the contrast agent around the ovary (bilateral) were more likely to be annular $(\mathrm{P}<0.05)$.

Conclusions: The imaging data gathered from TV 4D HyCoSy in TFI patients were comprehensive, which suggested that TV 4D HyCoSy could have potential to be used to assess the necessity of post-HyCoSy ART intervention in patients with TFI. This could be of benefit in reducing the incidence of overtreatment and potential complications of ART.

Keywords: Transvaginal 4-dimensional hysterosalpingo-contrast sonography (TV 4D HyCoSy); tubal factor infertility (TFI); assisted reproductive technology (ART); pregnancy

Submitted Oct 27, 2020. Accepted for publication Mar 20, 2021.

doi: $10.21037 /$ qims-20-1193

View this article at: http://dx.doi.org/10.21037/qims-20-1193 


\section{Introduction}

Evaluation of tubal patency and uterine characteristics is the first-step examination to assess infertility. Tubal factor infertility (TFI) refers to a type of infertility in which women cannot be fertile due to blockage, adhesion, and patency of the fallopian tubes (1). TFI is the most common cause of female infertility, accounting for $>30 \%$ of female infertility cases (2). Accurate evaluation of the patency, adjacent relationship, and function of the fallopian tubes is of foremost importance, not only for diagnosis but also for timely and precise treatment. Laparoscopic examination along with tubal chromopertubation dye test (LDT) and hysterosalpingography (HSG) was once accepted as the gold standard for such evaluation (3). However, in order to improve the assessment of tubal patency, in addition to the 2 traditional methods of LDT and HSG, innovative research has continued across the world. Some researchers have focused on diagnostic hysteroscopy (4), in which the evaluation is based on a straightforward view of a "flow" effect of hysteroscopic fluid in the fallopian tube ostia $(5,6)$. A prospective cohort study reported a sensitivity of $85.3 \%$ (95\% CI: $76-92 \%$ ) and a specificity of $66.1 \%$ (95\% CI: $52-78 \%)$ in predicting patency $(\mathrm{P}<0.001)(7)$. Although it obviates the potential risks of ionizing radiation and iodine allergy, diagnostic hysteroscopy does not seem suitable for screening due to the highly subjective evaluation, dependence on technician experience, and requirements of accessing the uterine cavity. Hysterosalpingo-contrastsonography (HyCoSy) has been a recent yet rapidly developing examination method. It has considerable application potential with the same or even better sensitivity and specificity than the preceding methods $(3,8,9)$.

The HyCoSy is performed by injection of foam and activation of grayscale ultrasound, identifying contrast flow in the tubal lumen and distinguishing it from surrounding tissue or from air in the bowels (10). Two-dimensional hysterosalpingo-contrast-sonography (2D HyCoSy) was first performed with the purpose of obviating ionizing radiation and the risk of iodine allergy, and has a sensitivity of $88 \%$ (95\% CI: $80-94 \%)$ and a specificity of $92 \%(95 \%$ CI: 87-95\%) according to one meta-analysis $(\mathrm{P}<0.05)(11)$. Additionally, it allows concomitant visualization of the ovaries and myometrium while avoiding ionizing radiation (12). However, due to the irregular course of the fallopian tube in the pelvic cavity, 2D HyCoSy rarely visualizes the entire fallopian tube and bilateral ovaries on the same plane. Furthermore, the first dose of contrast agent dispersed in the pelvic cavity often interferes with the contrast image. Three-dimensional imaging with HyCoSy (3D HyCoSy) was then proposed, displaying the $3 \mathrm{D}$ structure of the fallopian tube and further improving on the sensitivity of 2D HyCoSy (13). Nevertheless, the inability to observe the overflow of the contrast agent in real time remained. Notably, real-time 3D HyCoSy, or four-dimensional (4D) HyCoSy, was developed to overcome this limitation, and has shown great strength in the assessment of tubal function. 4D HyCoSy can visually display the entire process of contrast agent flowing from the uterine cavity to the fimbriae of fallopian tube on the basis of real-time data collection and reconstruction (14). Such dynamic imaging can offer more information about the course of the fallopian tube and its relationship to the surrounding areas. When the fallopian tube is not smooth, the obstructed part is characterized as tortuous and thick. Fallopian tube adhesions can also be determined by obstruction to the contrast stream, distension of the fallopian tube and overflow and dispersion condition of contrast agent around the ovary. (15). Furthermore, the ultrasound contrast agent has also been upgraded from negative contrast agents (normal saline) to positive contrast agents, including microbubble contrast agents, like SonoVue (Bracco, Milan, Italy) and hydroxyethylcellulose (16), which allow for the real-time observation of overflow of the contrast medium. The stability of the new microbubble is such that it can maintain an extended duration, allowing operators enough time for dynamic observation of multiple angles and planes and of the flow of contrast medium from the uterine cavity to the fallopian tubes and fimbriae (17). Similar to SonoVue, perfluoropropane-albumin microsphere is another kind of microbubble and has the advantages over SonoVue of not requiring metabolization in vivo and of providing good cell adsorption performance (18). Hence, the TV 4D HyCoSy is a non-invasive examination method with many advantages including easy operation, a lack of ionizing radiation, tolerable discomfort, low cost, and multi-sectional and multi-angular observation capacity, making it increasingly clinically favored in tubal patency assessment $(19,20)$.

The use of assisted reproductive technology (ART) has been proven in infertility treatments, and includes in vitro fertilization (IVF) and non-IVF. The former is the process by which an oocyte is fertilized by semen outside the body, while the latter involves ovulation induction, artificial insemination, and intrauterine insemination (IUI) $(21,22)$. Although the use of ART has become increasingly common, the cost and potential complications cannot be ignored or 
overlooked: both the mother and the fetus are susceptible to harm from ART. Numerous researches were devoted to identify non-IVF is safer than IVF. For instance, the occurrence of subchorionic hematoma (23), ischemic placental disease (24), placenta accreta spectrum (25), and neonatal complications (26) was less likely to happen in non-IVF. Nevertheless, there is no denying that ART has been reported to be associated with some undesirable outcomes. Regarding the fetus, ART-conceived infants were found to have a higher likelihood of birth defects in the cardiovascular, musculoskeletal, urogenital, gastrointestinal, and respiratory systems than spontaneously conceived infants $(27,28)$. For the mother, the latest research has reported that ART may induce pregnancy-related venous thromboembolism (VTE) (29), preeclampsia (30), and enhanced risk of breast cancer (31), and exert negative impacts on maternal postnatal mental health (32).

We conducted this research with the aim of developing a way to non-invasively, accurately, effectively, safely, and simply assess whether there is a likelihood of natural pregnancy in TFI patients, and if it is necessary to perform ART. In this study, we presented the differences between the 4D HyCoSy imaging data of women with TFI who eventually conceived. The comparison focused on the general conditions and imaging characteristics of those women who spontaneously conceived within 3 months following 4D HyCoSy and those who conceived after ART.

\section{Methods}

\section{Participants}

The study was conducted between March 1, 2017 and May 31,2018 . A total of 1,433 women with TFI were recruited at the Department of Obstetrics and Gynecology of Guangxiu Hospital (Changsha, Hunan, China). A complete medical history was taken, and each participant underwent TV 4D HyCoSy. All participants were provided with the appropriate information about the study objectives, particularly that which concerned the determination of pregnancy outcomes. The expected time for natural pregnancy was 3 months after the 4D HyCoSy examination. The follow-up period was 1 year. This study was conducted in accordance to the Declaration of Helsinki for medical research, and local approval was obtained from the ethics committee and the institutional review board (NO.2020-S579).

Infertility is characterized by the failure to achieve a clinical pregnancy after 12 months of regular unprotected sexual intercourse (33). The study included sexually active women in the reproductive age group of 20-45 years who were highly suspected of having TFI. These patients were previously found poor patency of the fallopian tubes through X-ray HSG or ultrasonography with complete clinical history data. Additionally, the body temperature had to be $<37.5^{\circ} \mathrm{C}$. Body temperature (BBT) was monitored for 3 consecutive months to ensure the participants with normal ovulatory function. Then, they were recommended to attempt spontaneous conception for $\geq 3$ months. There was a participant requirement for general good health in order to be able to tolerate the examination. Finally, only participants who provided written informed consent to be involved in the study were included.

Participants were excluded from the study if any of the following criteria were met: (I) contraindication for $\mathrm{HyCoSy}$, such as suspected pregnancy, malignant tumors of the genital tract, genital tract infections, or acute pelvic inflammatory; (II) presence of other factors responsible for infertility combined with TFI, such as congenital abnormalities in the genital tract, ovulation disorders, endometriosis, uterine fibroids, intrauterine adhesions, chromosomal abnormalities, or endocrine and immune factors; (III) history of unilateral or bilateral salpingectomy; (IV) poor compliance and poor cooperation during the examination; (V) severe drug allergies, and severe systemic illness or mental illness; (VI) grossly abnormal semen in male partner.

\section{Performance of $4 \mathrm{D} \mathrm{HyCoSy}$}

A Voluson E8 ultrasound system with coded contrast imaging (GE Healthcare, Milwaukee, WI, USA) was used. The mechanical index of the instrument was set at 0.12-0.18. An RIC5-9-D transvaginal volume transducer (GE Healthcare, Milwaukee, WI, USA) with a frequency of $5.0-7.5 \mathrm{MHz}$ and scanning angles of $0-179^{\circ}$ was used in the examinations.

Perfluoropropane-albumin microsphere injection (Kangrun Pharmaceutical Co., Yueyang, Hunan, China) was used to prepare the contrast medium for 4D HyCoSy. More precisely, the content of 1 perfluoropropane-albumin microsphere injection vial was a $3 \mathrm{~mL}$ suspension which was then diluted with $17 \mathrm{~mL}$ of $0.9 \%$ normal saline. The diluted solution of perfluoropropane-albumin microsphere injection was then used in the HyCoSy examination.

4D HyCoSy was conducted within 3-7 days following menstruation. Patients reclined on a gynecological chair in 
the dorsal lithotomy position. To prevent tubal spasms, all patients received an intramuscular injection of anisodamine $30 \mathrm{~min}$ before the procedure. After sterilizing the vagina, a 12-Fr Foley catheter (Zhanjiang Star Enterprise Co., LTD., Guangdong, China) was inserted into the uterine cavity. The balloon of the catheter was filled with $0.6-4 \mathrm{~mL}$ of $0.9 \%$ normal saline at $37{ }^{\circ} \mathrm{C}$ according to the degree of cervical relaxation and withdrawn to ensure the obstruction of the internal orifice of the uterus and of the cervical canal. Transvaginal sonography (TVS) was performed first to observe the shape, size, and peripheral adjacency of the uterus and ovary, to determine whether there was adhesion, and to detect the presence of free liquid areas in the pelvis. Concurrently, the most favourably exposed section of bilateral uterine horns was found, and then 3D scanning was performed in an attempt to display the bilateral uterine horns and bilateral ovaries within the same 3D scan area. Then, the ultrasound system was set to the contrast mode and the 4D scanning mode was activated with the appropriate gain depth and focus range to achieve the best image. The uterine cavity was filled with $20 \mathrm{~mL}$ of prepared contrast agent through the catheter, and the size of the balloon was adjusted according to the pressure. Real-time tracking of the proximal, middle, and distal fimbria of the bilateral fallopian tubes was performed, so that the position of the segments of intrauterine cavity and fallopian tubes in the pelvic cavity were clearly displayed. Meanwhile, the relationship between the ovary and the diameter, position, and distal fimbria of the fallopian tubes, in addition to the flow and overflow of the contrast agent around the ovary, was dynamically observed (Figure 1). The final step was to withdraw the solution from the balloon and remove the catheter. After the procedure, Magic CUT, a computer software, was used to trim the image, to show clear images of the uterine cavity and fallopian tubes, and to analyze the dynamic image and determine the degree of tubal patency. The examinations and evaluation reports were performed by 5 sonographers, each of whom had $>5$ years of experience using ultrasonography to perform gynecologic and obstetric examinations.

\section{Parameters studied}

We collected basic clinical details of the participants including age, infertility type, and infertility duration. We documented the basic performance data including length of the balloon, dosage of the contrast agent, volume of the balloon, and reflux, resistance and reflux condition. We further analyzed the imaging data including the endometrial thickness, the position of the ovaries and uterus, ovarian motility, the fallopian tube visualization and morphology, the intactness of the fallopian tube wall, fallopian tube smoothness, the overflow condition of the contrast agent from the fimbriae of fallopian tube, the $0 / 1$ dispersion of the contrast agent around the ovaries, and the infiltration of the contrast agent into the myometrium during TV 4D $\mathrm{HyCoSy}$.

\section{Statistical analysis}

Statistical analysis was performed with SAS software version 9.4 (Statistical analysis system, SAS Institute Inc., Cary, NC, USA). Differences between participants with spontaneous conception and those with ART were tested using either a chi-squared $\left(\chi^{2}\right)$ or Fisher's exact test as appropriate. Stepwise regression analysis was applied to determine a single dominant factor. A P value $<0.05$ was considered statistically significant.

\section{Results}

After applying the inclusion and exclusion criteria and waiting 3 months for spontaneous conception and 1 year for the follow-up, a total of 1,085 patients of the 1,433 patients were followed up for pregnancy outcomes in our hospital's Obstetrics and Gynecology Department. No further treatment was performed after 4D HyCoSy. Among the 1,085 patients, 301 patients had spontaneously conceived (27.74\%), and 405 patients had successfully conceived after ART $(37.33 \%)$ (Figure 2).

Through the statistical analysis of the relevant clinical data of 706 participants who conceived after TV 4D HyCoSy, it was revealed that age, infertility duration, endometrial thickness, ovarian motility, fallopian tube visualization, overflow condition of contrast agent from fimbriae of the fallopian tube, $0 / 1$ dispersion of the contrast agent around the ovaries, resistance to the injection of the contrast agent, and reflux condition of the contrast agent were significantly related to postoperative spontaneous conception $(\mathrm{P}<0.05)$. Other variables were not statistically significant $(\mathrm{P}>0.05)$ (Table 1).

Univariate analysis evaluated the factors associated with ART necessity (Table 2). Compared with the natural pregnancy group, the ART group was composed of women who were older $[\mathrm{P}<0.05$, odds ratio $(\mathrm{OR}) 1.04,95 \% \mathrm{CI}$ : 1.01-1.08], with longer infertility duration $(\mathrm{P}<0.001$, 

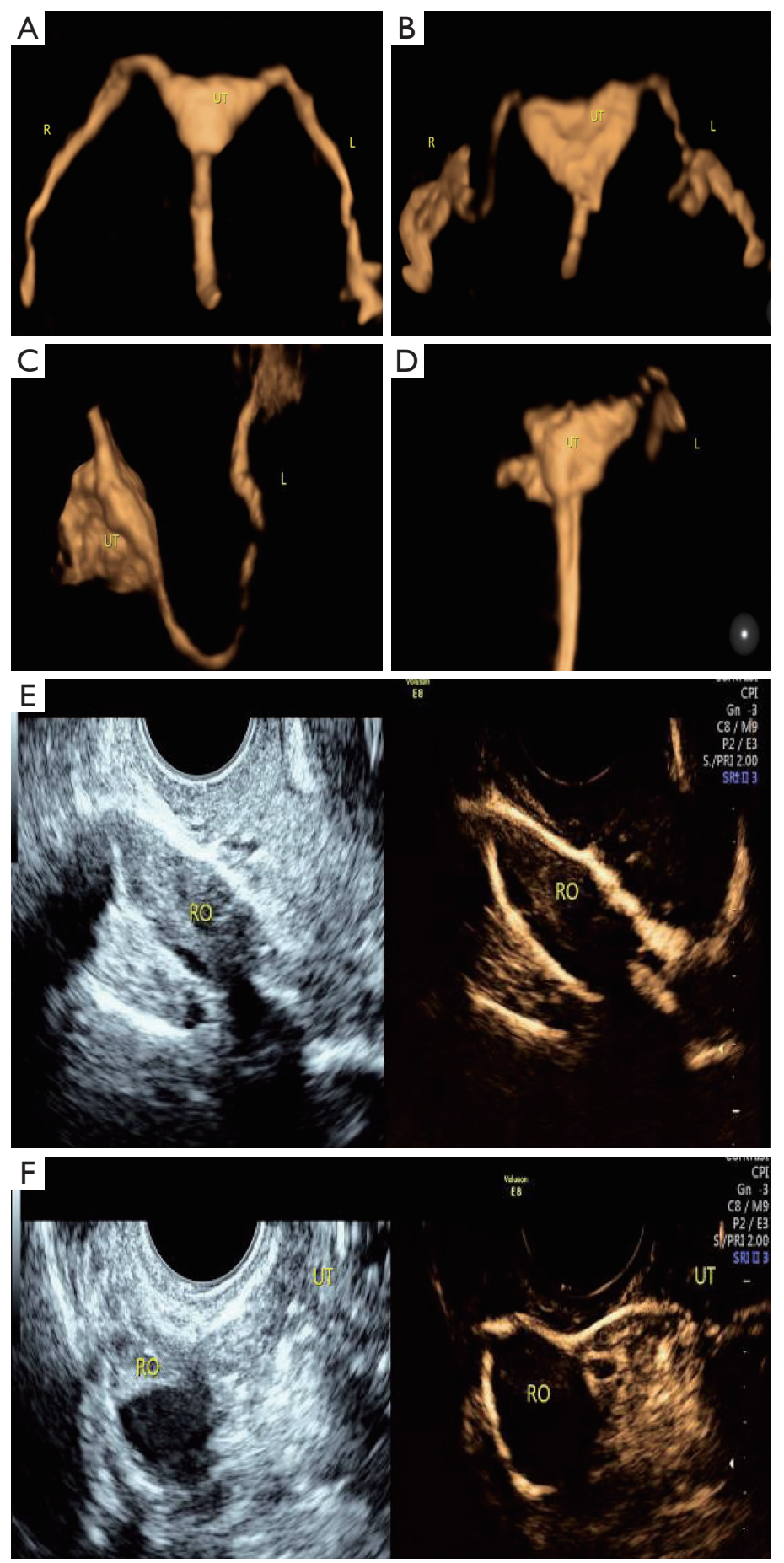

Figure 1 Typical characteristics of fallopian tubes on 4D HyCoSy. (A) Bilateral fallopian tubes with good patency: the contrast agent can smoothly flow into the fallopian tube and sprayed at the fimbria of the tube. The passage of the tube was soft. (B) Bilateral fallopian tubes with poor patency: the entire fallopian tube and the spillage at the fimbria of the tube were visible, but the passage of the tube was stiff and filamented. (C) The left tube with poor patency: the left tube was discontinuous, and the passage of the tube was angled, circuitous, and directed upward. (D) Bilateral fallopian tubes with obstruction: both fallopian tubes were obstructed, and the entire passage of the tubes or the spillage at the fimbria of the tube is invisible. (E) The dispersion of contrast agent around the right ovary shaped annular. (F) The dispersion of contrast agent around the right ovary shaped semi-annular. 


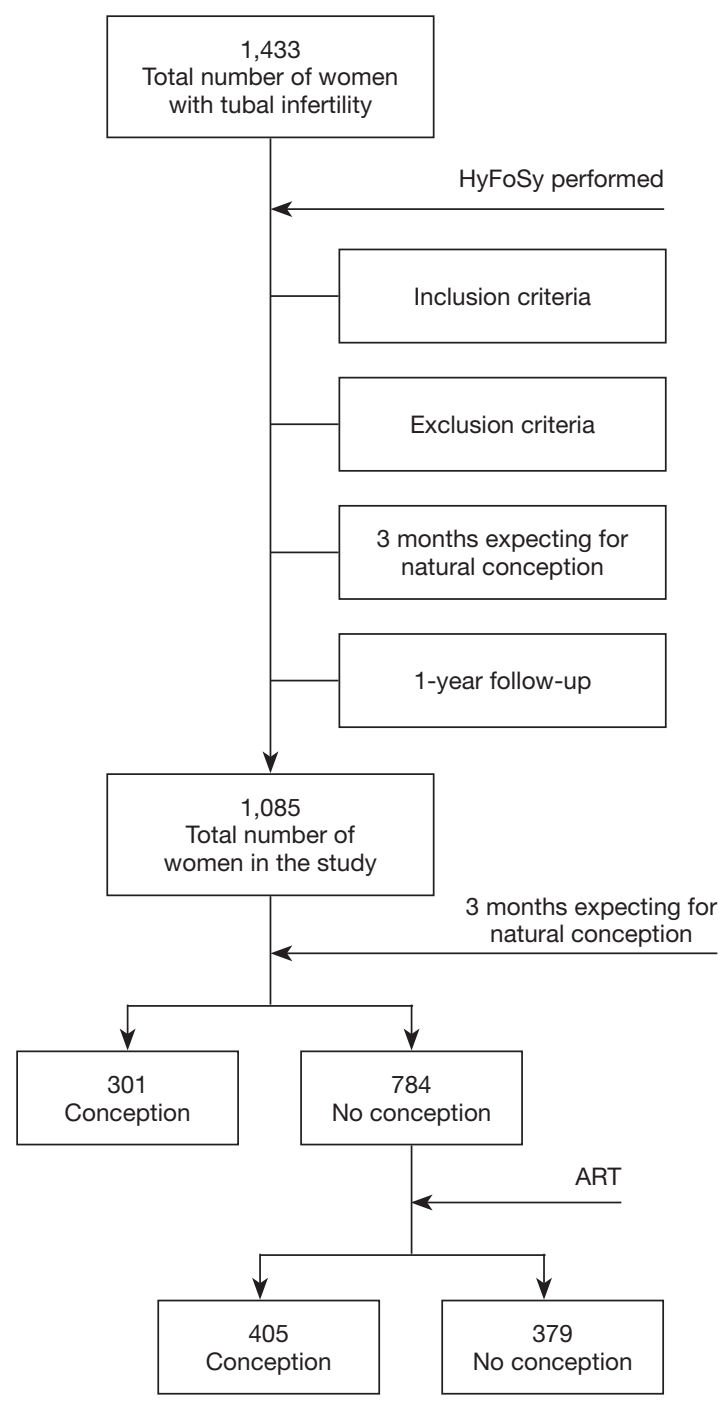

Figure 2 Flowchart of study participants.

OR 1.12, 95\% CI: 1.05-1.19), and thicker endometrium $(\mathrm{P}<0.01$, OR 1.13, 95\% CI: 1.04-1.22). Participants who used ART were more likely to display (I) limited ovarian motility, (II) poor fallopian tube visualization, (III) incomplete fallopian tube wall, (IV) rough morphology of the right fallopian tube, $(\mathrm{V})$ unsatisfactory contrast agent overflow condition, (VI) dispersion of contrast agent around ovaries, and (VII) high resistance and reflux (resistance in the latter: $\mathrm{P}<0.01$, OR 2.06, 95\% CI: 1.21-3.49; persistent resistance: $\mathrm{P}<0.001$, OR 2.08, 95\% CI: 1.45-2.99; reflux condition: $\mathrm{P}<0.01$, OR $1.51,95 \%$ CI: $1.12-2.04)$ during the procedure. Other variables showed no difference between the 2 groups $(\mathrm{P}>0.05)$.

Multivariate analysis using logistic regression was used to identify independent factors influencing the method of conception. The regression model was built based on stepwise regression methods. We identified the most significant predictive factors (meaningful variables whose $\mathrm{P}$ values $<0.05$ ) for the necessity of ART in infertility patients (Table 3). Overall, our results indicated that age, infertility duration, endometrial thickness, and poor $0 / 1$ dispersion of the contrast agent around the ovaries were factors that increased the probability of required ART, while the grade of bilateral fallopian tube visualization and the grade of the contrast agent overflow condition were revealed as the likely protective factors.

\section{Discussion}

This study was first to explore whether the necessity of ART can be evaluated with the assistance of 4D HyCoSy. Couples with fertility problems can still have early and non-evidence-based access to ART in order to save time with respect to the woman's reproductive window (34). It is undeniable that after $>50$ years of technological improvement, ART has gradually taken the place of drug therapy as a reliable and effective treatment for infertile women. For TFI nulliparous women, the ART success rate has demonstrated no statistical difference in patients with a history of ectopic pregnancy or intrauterine pregnancy (35). However, the application of ART requires critical evaluation because it still has the risk of increasing antenatal and perinatal complications, and poor neonatal outcomes in the short term $(36,37)$. If certain non-invasive exams can give TFI women who have good potential for spontaneous conception a chance, complications from ART can be avoided in larger numbers. Transvaginal 4D HyCoSy is a reliable method for standard reference. In addition to its sensitivity to tubal function, it can also detect specific uterine diseases that are closely related to infertility such as Mullerian malformation, myomas, endometrial polyps, and Asherman's syndrome.

Our study involved 1,085 infertile women with TFI, 706 of whom conceived, with $27.74 \%$ of these conceiving spontaneously in 3 months following 4D HyCoSy and $37.33 \%$ after ART. On the one hand, the sample size was relatively reasonable for a pilot study; on the other hand, the procedure was found to be safe and without any drawbacks such as pain, infection, or intolerance. In contrast to transabdominal ultrasound, TV 4D HyCoSy not only avoids having to drink copious amounts of water and retain urine, but also avoids the adverse effects of abdominal 
Table 1 Clinical characteristics of pregnant women after TV 4D HyCoSy

\begin{tabular}{|c|c|c|c|}
\hline Variates & Spontaneously conceived & ART & P-value \\
\hline Age & $30.18(4.20)$ & $30.99(4.61)$ & $<0.05$ \\
\hline Infertility duration (years), mean (SD) & $2.68(2.17)$ & $3.42(2.92)$ & $<0.001$ \\
\hline Endometrial thickness (mm), mean (SD) & $6.69(1.86)$ & $7.14(2.01)$ & $<0.01$ \\
\hline Dosage of contrast agent (mL), mean (SD) & $0.92(0.41)$ & $0.94(0.49)$ & 0.45 \\
\hline Volume of balloon (mL), mean (SD) & $18.73(4.86)$ & $18.24(6.19)$ & 0.256 \\
\hline Volume of reflux (mL), mean (SD) & $2.39(4.55)$ & $2.83(4.46)$ & 0.2 \\
\hline \multicolumn{4}{|l|}{ Number (\%) } \\
\hline 1-Distant & $78(25.9)$ & $122(30.2)$ & \\
\hline Position of right ovary and uterus, n (\%) & & & 0.897 \\
\hline \multicolumn{4}{|l|}{ 0-Adjacent } \\
\hline 1-Distant & $129(42.9)$ & $170(42.1)$ & \\
\hline Number of ovaries far from the uterus, $\mathrm{n}(\%)$ & & & 0.373 \\
\hline 0 & $131(43.5)$ & $158(39.1)$ & \\
\hline 1 & $133(44.2)$ & $200(49.5)$ & \\
\hline 2 & $37(12.3)$ & $46(11.4)$ & \\
\hline \multicolumn{4}{|l|}{ 0-Good } \\
\hline \multicolumn{4}{|l|}{ 0-Good } \\
\hline 1-Poor & $110(36.5)$ & $209(51.7)$ & \\
\hline Number of ovaries with limited motility, $\mathrm{n}(\%)$ & & & $<0.001$ \\
\hline 0 & $122(40.5)$ & $97(24.0)$ & \\
\hline 1 & $93(30.9)$ & $144(35.6)$ & \\
\hline 2 & $86(28.6)$ & $164(40.3)$ & \\
\hline Left fallopian tube visualization, $\mathrm{n}(\%)$ & & & $<0.001$ \\
\hline 0-Entire & $263(87.4)$ & $315(77.7)$ & \\
\hline 1-Intermittent & $33(11.0)$ & $60(14.9)$ & \\
\hline $2-\mathrm{No}$ & $5(1.7)$ & $30(7.4)$ & \\
\hline
\end{tabular}

Table 1 (continued) 
Table 1 (continued)

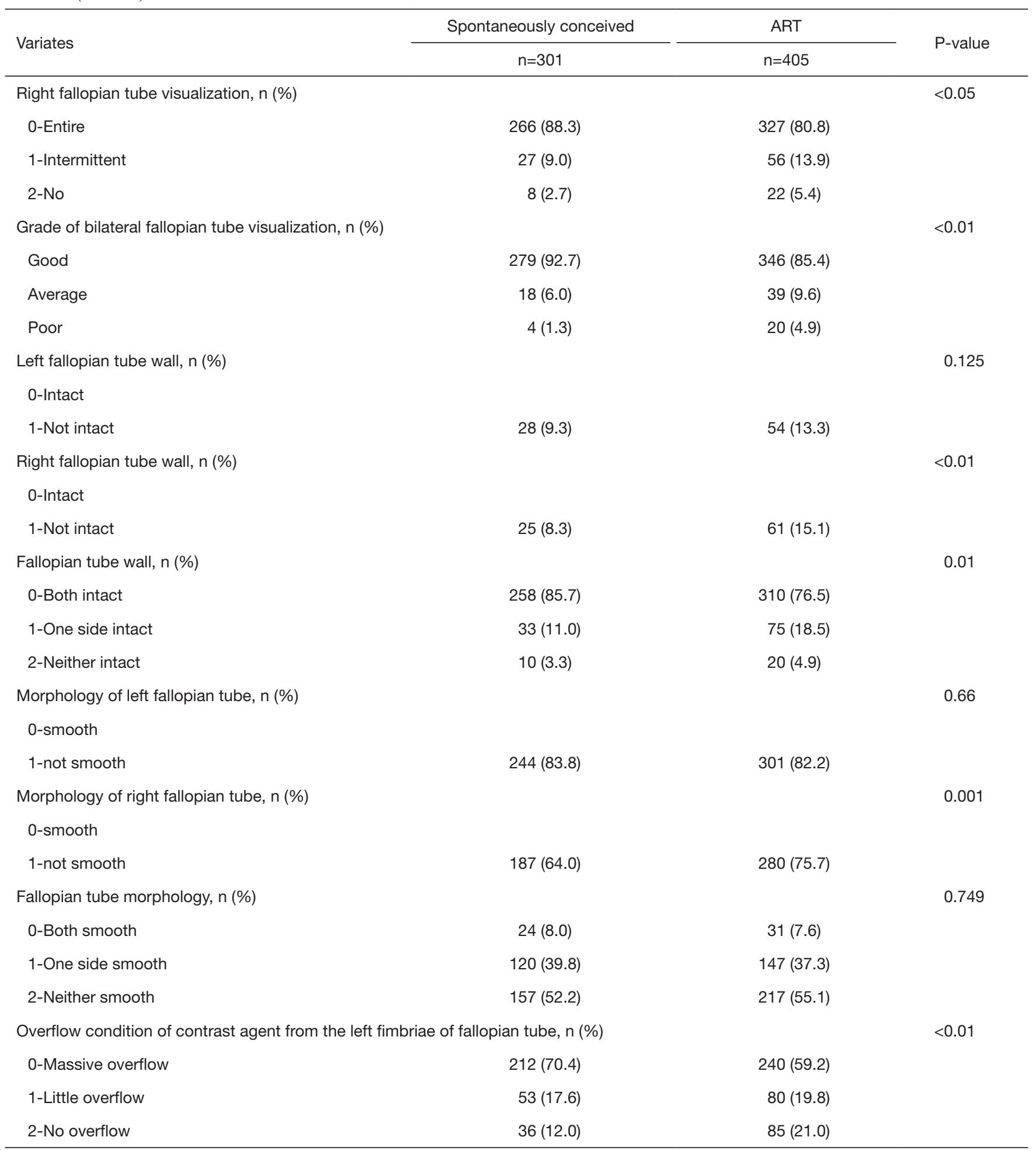

Table 1 (continued) 
Table 1 (continued)

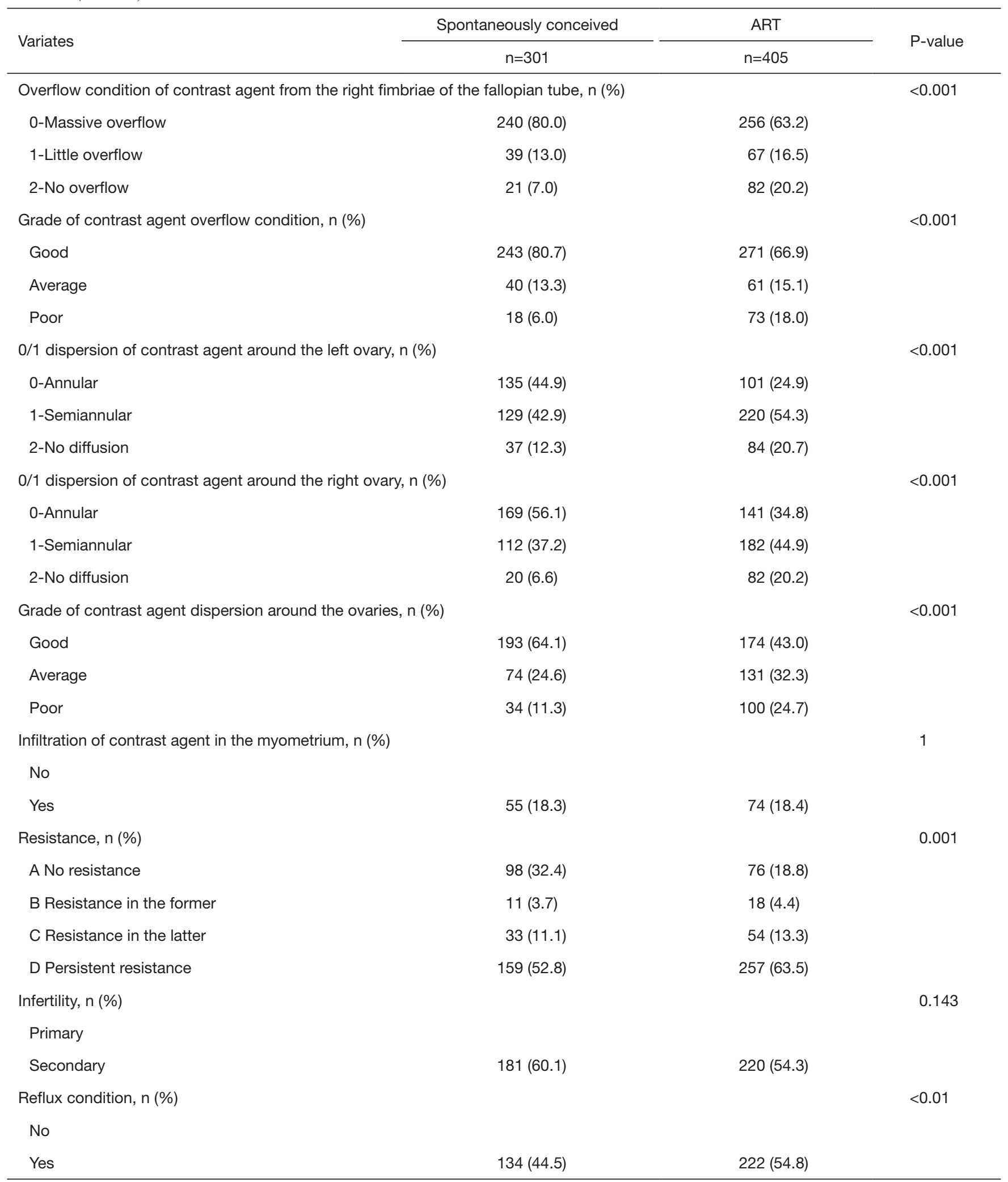

TV 4D HyCoSy, transvaginal 4-dimensional hysterosalpingo-contrast-sonography. 
Table 2 Univariate analysis of ART necessity

\begin{tabular}{|c|c|c|c|c|c|c|}
\hline Variates & Estimate & Std. error & $z$ value & $\mathrm{P}$ & OR & $95 \% \mathrm{Cl}$ \\
\hline Infertility duration (years) & 0.1143 & 0.0318 & 3.5918 & $<0.001$ & 1.12 & $1.05-1.19$ \\
\hline Endometrial thickness (mm), mean (SD) & 0.1199 & 0.04 & 2.9995 & $<0.01$ & 1.13 & $1.04-1.22$ \\
\hline Length of balloon (cm) & -0.0514 & 0.0388 & -1.323 & 0.1858 & 0.95 & $0.88-1.03$ \\
\hline Volume of balloon (mL) & 0.1278 & 0.1691 & 0.7557 & 0.4498 & 1.14 & $0.82-1.58$ \\
\hline Volume of reflux (mL) & 0.0224 & 0.0176 & 1.2763 & 0.2019 & 1.02 & $0.99-1.06$ \\
\hline \multicolumn{7}{|l|}{ Position of the left ovary and uterus } \\
\hline \multicolumn{7}{|l|}{ 0-Adjacent } \\
\hline \multicolumn{7}{|l|}{ 0-Adjacent } \\
\hline 1-Distant & -0.0318 & 0.154 & -0.2067 & 0.8362 & 0.97 & $0.72-1.31$ \\
\hline \multicolumn{7}{|l|}{ Number of ovaries far from the uterus } \\
\hline \multicolumn{7}{|l|}{0} \\
\hline 1 & 0.2206 & 0.1627 & 1.3554 & 0.1753 & 1.25 & $0.91-1.72$ \\
\hline 2 & 0.0303 & 0.2505 & 0.1211 & 0.9036 & 1.03 & $0.63-1.68$ \\
\hline \multicolumn{7}{|l|}{ Left ovarian motility } \\
\hline \multicolumn{7}{|l|}{ 0-Good } \\
\hline \multicolumn{7}{|l|}{0} \\
\hline 1 & 0.6665 & 0.1903 & 3.503 & $<0.001$ & 1.95 & $1.34-2.83$ \\
\hline 2 & 0.8687 & 0.1904 & 4.5615 & $<0.001$ & 2.38 & $1.64-3.46$ \\
\hline \multicolumn{7}{|l|}{ Left fallopian tube visualization } \\
\hline \multicolumn{7}{|l|}{ 0-Entire } \\
\hline 1-Intermittent & 0.4206 & 0.2323 & 1.8107 & 0.0702 & 1.52 & $0.97-2.4$ \\
\hline 2-No & 1.6145 & 0.4902 & 3.2934 & 0.001 & 5.03 & $1.92-13.14$ \\
\hline \multicolumn{7}{|l|}{ Right fallopian tube visualization } \\
\hline \multicolumn{7}{|l|}{ 0-Entire } \\
\hline 1-Intermittent & 0.5229 & 0.2492 & 2.0981 & $<0.05$ & 1.69 & $1.04-2.75$ \\
\hline 2-No & 0.7765 & 0.4237 & 1.8327 & 0.0669 & 2.17 & $0.95-4.99$ \\
\hline
\end{tabular}

Table 2 (continued) 
Table 2 (continued)

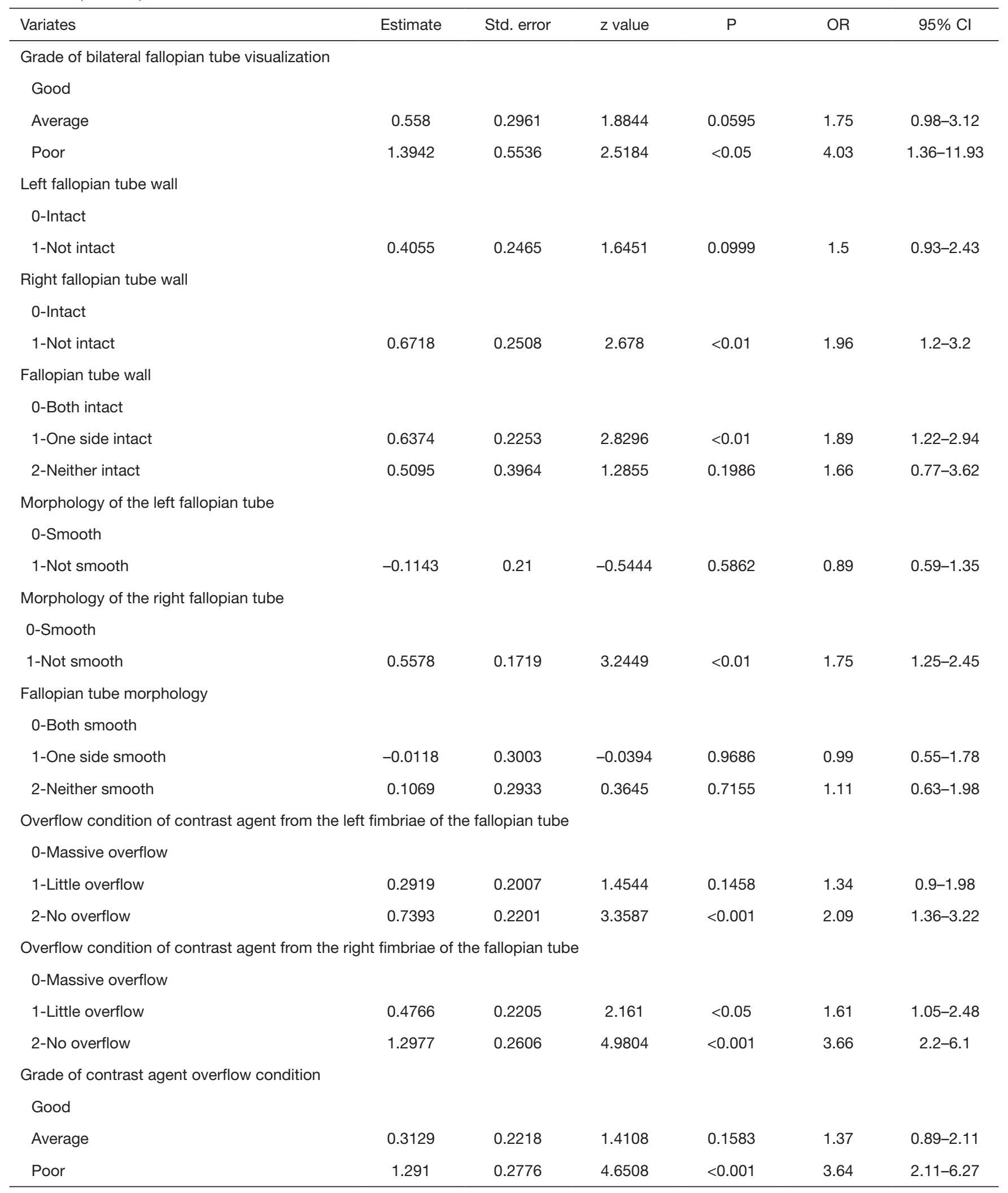

Table 2 (continued) 
Table 2 (continued)

\begin{tabular}{|c|c|c|c|c|c|c|}
\hline Variates & Estimate & Std. error & $z$ value & $\mathrm{P}$ & OR & $95 \% \mathrm{Cl}$ \\
\hline \multicolumn{7}{|l|}{ 0-Annular } \\
\hline 1-Semiannular & 0.824 & 0.1721 & 4.7888 & $<0.001$ & 2.28 & $1.63-3.19$ \\
\hline 2-No diffusion & 1.1101 & 0.2372 & 4.6808 & $<0.001$ & 3.03 & $1.91-4.83$ \\
\hline \multicolumn{7}{|l|}{ 0-Annular } \\
\hline 1-Semiannular & 0.6666 & 0.1656 & 4.025 & $<0.001$ & 1.95 & $1.41-2.69$ \\
\hline 2-No diffusion & 1.5921 & 0.2742 & 5.8057 & $<0.001$ & 4.91 & $2.87-8.41$ \\
\hline \multicolumn{7}{|c|}{ Grade of contrast agent dispersion around the ovaries } \\
\hline Poor & 1.1824 & 0.2244 & 5.2702 & $<0.001$ & 3.26 & $2.1-5.06$ \\
\hline \multicolumn{7}{|c|}{ Infiltration of contrast agent in the myometrium } \\
\hline \multicolumn{7}{|l|}{ No } \\
\hline Yes & 0.0019 & 0.197 & 0.0098 & 0.9922 & 1 & $0.68-1.47$ \\
\hline Resistance & & & & 0.1092 & & \\
\hline \multicolumn{7}{|l|}{ A No resistance } \\
\hline B Resistance in the former & 0.7393 & 0.4126 & 1.792 & 0.0731 & 2.09 & $0.93-4.7$ \\
\hline C Resistance in the latter & 0.7206 & 0.27 & 2.6687 & $<0.01$ & 2.06 & $1.21-3.49$ \\
\hline \multicolumn{7}{|l|}{ Infertility } \\
\hline \multicolumn{7}{|l|}{ Reflux condition } \\
\hline \multicolumn{7}{|l|}{ No } \\
\hline Yes & 0.4133 & 0.153 & 2.701 & $<0.01$ & 1.51 & $1.12-2.04$ \\
\hline
\end{tabular}

ART, assisted reproduction technology.

fat thickness on final imaging. In this study, the main differences of the general condition between the 2 groups were age, infertility duration, and endometrial thickness. Age and duration of infertility are known to be significant predictors for potential spontaneous conception; younger women with a shorter duration of infertility are more likely to conceive without ART (38). Meanwhile, studies have also shown that age and duration of infertility are important factors affecting the rate of assisted reproduction in women with TFI (39). However, when it comes to endometrial thickness, which may reflect endometrial receptivity (40). It is generally believed that a thicker endometrium facilitates embryo implantation, and one study indicates that an endometrial thickness $>12 \mathrm{~mm}$ is a strong protective factor against ectopic pregnancy (41). Nevertheless, other investigators have also reported that a thicker endometrium may result in a low pregnancy rate (42), and a very recent study reported that the pregnancy rate after frozen-thawed embryo transfer was significantly decreased in patients whose endometrium was $>7 \mathrm{~mm}$ compared with those 
Table 3 Stepwise logistic regression of variables in the natural pregnancy group and assisted reproduction group

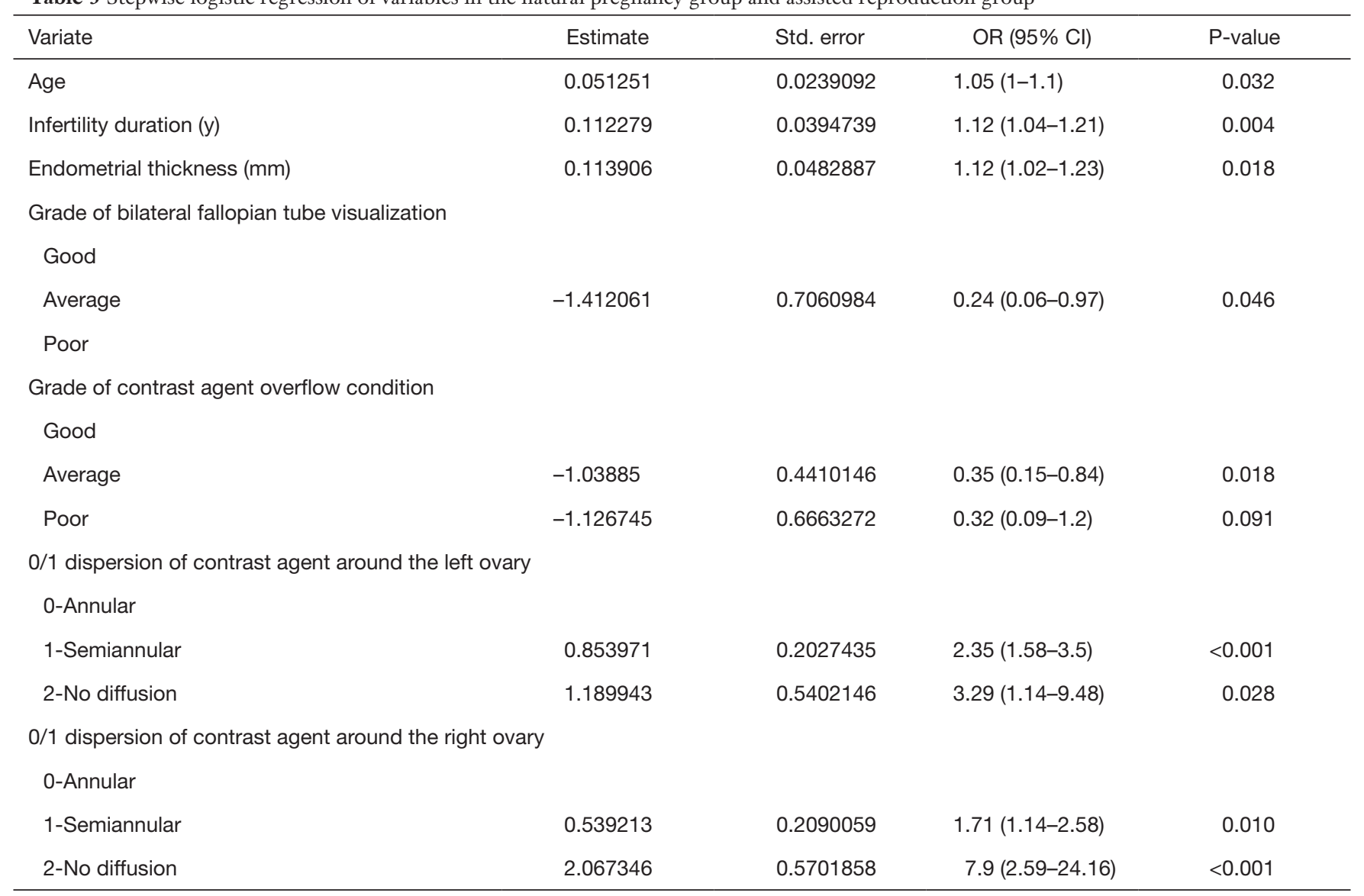

OR, odds ratio; $\mathrm{Cl}$, confidence interval.

whose endometrium was $<7 \mathrm{~mm}$ (43). In our study, the endometrium of the natural pregnancy group was thinner, with an average of $6.69 \mathrm{~mm}$, and the ART group had a thicker endometrium average of $7.14 \mathrm{~mm}$. This may prove that the thickness of the endometrium is not the only criterion for embryo implantation or could be related to the imaging error caused by contrast agent intravasation into the uterine myometrium and venous plexus (14). In patients with tubal pathology in particular, fallopian tube obstruction will induce difficult fallopian tube flow, and the injection pressure of the contrast agent will be higher, leading to higher penetration of the contrast agent and an incorrect endometrial thickness reading.

The most important observation of our study is that TV 4D HyCoSy is fast, creates clear images, and can dynamically reflect the structure and shape of the fallopian tube in real time, which provides more subdivided and detailed examination results for evaluating fallopian tube function. Among these, fallopian tube visualization, overflow condition of contrast agent from the fimbriae of fallopian tube, resistance, contrast agent injection resistance, and reflux condition can be applied to evaluate tubal patency from multiple angles. In terms of injection resistance, only resistance at the last moments and persistent resistance were significant, which is consistent with research which has confirmed that the implementation of IVF instead of IUI may be a more appropriate approach for patients with unilateral distal tubal blockage (44). Interestingly, we found that when it comes to fallopian tube visualization and morphology, and intactness of the fallopian tube wall, importance is only attached to the right side. As has been previously reported, the right ovary contains more follicles than the left ovary (45). The frequency of ovulation occurring in the right side is significantly higher than that of the left side, and oocytes from the right ovary are more likely to cause pregnancy with no statistical difference between normal and infertile women (46). In IVF, the response of the right ovary to follicle-stimulating hormone 
(FSH) is better than that of the left (47). This difference can be explained by anatomy: the connection between the left ovarian and renal veins is perpendicular, and thus the blood reflux and the temperature in the left ovary is greater, minimizing the fertility potential of the oocytes in the left ovary. Xia et al. reported that patients had a rightsided lateral dominance for tubal pregnancy, which is in accordance with our study (48). They also found a tendency that the proportion of right-sided tubal pregnancies decreased with age, and patients in the age group $<40$ years had more right-sided tubal pregnancies (48). In addition, the ovarian mobility and dispersion of contrast agent around the right ovary are also important risk factors. These indicators may indirectly characterize the adhesions around the pelvis. Pelvic adhesion has a close relationship with infertility due to hydrosalpinx and tube distortion which makes the possibility for proximal tubal recanalization lower than normal $(49,50)$.

Besides its diagnostic value, HyCoSy has therapeutic value in being able to flush contrast agent (51). Fluid passage through the tubal lumen can flush inspissated mucus plugs and remove minor adhesions or buildup material caused by inflammatory processes $(16,52)$. Many studies have reported higher fecundity rates for several months after the procedure (53). In one study, HyCoSy's beneficial effect was observed directly after the procedure, and an increased pregnancy rate of up to $45 \%$ was observed in the first 30 days after HyCoSy $(\mathrm{P}<0.0005)$ (54). One systematic review found that the use of an oil-based contrast agent was associated with increased pregnancy and live birth rates compared to the different outcomes of other contrast agents (51). However, this view is still controversial, and a large-scale prospective study is needed for verification (55). Nevertheless, there is no doubt that using the contrast agent, perfluoropropane-albumin microsphere in TV 4D HyCoSy not only plays a part in flushing and dredging the fallopian tube, but can also improve blood supply and promote the absorption of inflammation, while the use of an oil-based contrast agent may impose potential negative effects on thyroid function and neurodevelopment $(56,57)$. Overall, TV 4D HyCoSy is a safe inspection method that does not impinge on pregnancy and fetal outcomes (58).

\section{Strengths and Limitations of the Study}

This study was the first to analyze the necessity of ART with the assistance of 4D HyCoSy. Also, we provided the largest sample size in 4D HyCoSy related studies so far. In addition, the key strength of this study lied in the first try to subdivide the 4D HyCoSy results to understand whether each subtle imaging parameter affects the prognosis. This greatly provided the basis for clinical judgment. The limitation of the study is that the TFI patients in our study did not make a diagnosis through the standard technology such as laparoscopic chromotubation. However, the accuracy of 4D HyCoSy itself has been reported to be very high (14) and the original intention of this study was to apply the relatively non-invasive methods and reduce injuries.

\section{Conclusions}

TV 4D HyCoSy is a non-invasive, accurate approach with the advantages of short inspection time, high repeatability, and low cost to perform. Given these traits, it accords with the most fundamental principle of medicine- "first, do no harm" - and can aid in both diagnosis and treatment. Its high sensitivity and accuracy make it a common imaging technique for infertility screening and an indirect assessment tool for fallopian tubal function in current clinical practice. Using the technique described above, detailed data can be documented and used to assess the possibility of natural pregnancy and the need for ART. In this way, the overtreatment and potential complications of ART can be avoided.

\section{Acknowledgments}

Funding: None.

\section{Footnote}

Conflicts of Interest: All authors have completed the ICMJE uniform disclosure form (available at http://dx.doi. org/10.21037/qims-20-1193). The authors have no conflicts of interest to declare.

Ethical Statement: The authors are accountable for all aspects of the work in ensuring that questions related to the accuracy or integrity of any part of the work are appropriately investigated and resolved. The trial was conducted in accordance with the Declaration of Helsinki (as revised in 2013). The study was approved by the institutional review board (IRB) of the Third Xiangya Hospital, Central South University (No. 2020-S579), and informed consent was taken from all individual participants.

Open Access Statement: This is an Open Access article 
distributed in accordance with the Creative Commons Attribution-NonCommercial-NoDerivs 4.0 International License (CC BY-NC-ND 4.0), which permits the noncommercial replication and distribution of the article with the strict proviso that no changes or edits are made and the original work is properly cited (including links to both the formal publication through the relevant DOI and the license). See: https://creativecommons.org/licenses/by-nc-nd/4.0/.

\section{References}

1. Dun EC, Nezhat CH. Tubal factor infertility: diagnosis and management in the era of assisted reproductive technology. Obstet Gynecol Clin North Am 2012;39:551-66.

2. Yuan L, Jingying H, Xiujuan C, Chengying L, Xiaochen H, Xiumei X, Yulong Z, Zihua C. Predictive value of a modified classification of fallopian tube status on prognosis of tubal factor infertility after laparoscopic surgery. Medicine (Baltimore) 2019;98:e14952.

3. Feinberg EC. True, true, and unrelated: tubal patency, tubal architecture, and tubal function. Fertil Steril 2018;110:646-7.

4. Promberger R, Simek IM, Nouri K, Obermaier K, Kurz C, Ott J. Accuracy of Tubal Patency Assessment in Diagnostic Hysteroscopy Compared with Laparoscopy in Infertile Women: A Retrospective Cohort Study. J Minim Invasive Gynecol 2018;25:794-9.

5. Hager M, Ott J, Holzer I, Seemann R, Kurz C, Parry JP. Hysteroscopic Assessment of Tubal Patency: A Randomized Comparison between the Flow and Parryscope Techniques. J Minim Invasive Gynecol 2020;27:1552-7.e1.

6. Hager M, Simek IM, Promberger R, Ott J. The Role of Diagnostic Hysteroscopy in the Evaluation of Fallopian Tube Patency: a Short Review. Geburtshilfe und Frauenheilkunde 2019;79:483-6.

7. Ott J, Hager M, Nouri K, Marschalek J, Kurz C. Assessment of Tubal Patency: A Prospective Comparison of Diagnostic Hysteroscopy and Laparoscopic Chromopertubation. J Minim Invasive Gynecol 2020;27:135-40.

8. Szamatowicz M, Szamatowicz J. Proven and unproven methods for diagnosis and treatment of infertility. Adv Med Sci 2020;65:93-6.

9. He Y, Ma X, Xu J, Li S, Wu H, Liu Q, Kong L, Luo J, Liu H. Comparison of Assessment Methods for Fallopian Tubal Patency and Peritubal Adhesion Between Transvaginal 4-Dimensional Hysterosalpingo-Contrast
Sonography and Laparoscopic Chromopertubation. J Ultrasound Med 2017 Mar;36:547-56.

10. Nannini R, Chelo E, Branconi F, Tantini C, Scarselli GF. Dynamic echohysteroscopy: a new diagnostic technique in the study of female infertility. Acta Eur Fertil 1981;12:165-71.

11. Maheux-Lacroix S, Boutin A, Moore L, Bergeron ME, Bujold E, Laberge P, Lemyre M, Dodin S.

Hysterosalpingosonography for diagnosing tubal occlusion in subfertile women: a systematic review with metaanalysis. Hum Reprod 2014;29:953-63.

12. Mandia L, Personeni C, Antonazzo P, Angileri SA, Pinto A, Savasi V. Ultrasound in Infertility Setting: Optimal Strategy to Evaluate the Assessment of Tubal Patency. Biomed Res Int 2017;2017:3205895-.

13. Alcázar JL, Martinez-Astorquiza Corral T, Orozco R, Dominguez-Piriz J, Juez L, Errasti T. Three-Dimensional Hysterosalpingo-Contrast-Sonography for the Assessment of Tubal Patency in Women with Infertility: A Systematic Review with Meta-Analysis. Gynecol Obstet Invest 2016;81:289-95.

14. Wang W, Zhou Q, Gong Y, Li Y, Huang Y, Chen Z. Assessment of Fallopian Tube Fimbria Patency With 4-Dimensional Hysterosalpingo-Contrast Sonography in Infertile Women. J Ultrasound Med 2017;36:2061-9.

15. Wang W, Zhou Q, Zhou X, Chen Z, Zhang H. Influence Factors on Contrast Agent Venous Intravasation During Transvaginal 4-Dimensional Hysterosalpingo-Contrast Sonography. J Ultrasound Med 2018;37:2379-85.

16. Exacoustos C, Pizzo A, Lazzeri L, Pietropolli A, Piccione E, Zupi E. Three-Dimensional Hysterosalpingo Contrast Sonography with Gel Foam: Methodology and Feasibility to Obtain 3-Dimensional Volumes of Tubal Shape. J Minim Invasive Gynecol 2017;24:827-32.

17. Zou P, Li M, Wang Z, Zhang G, Jin L, Pang Y, Du L, Duan Y, Liu Z, Shi Q. Micro-Particle Image Velocimetry Investigation of Flow Fields of SonoVue Microbubbles Mediated by Ultrasound and Their Relationship With Delivery. Front Pharmacol 2020;10:1651.

18. Hong Q, Cai R, Chen Q, Zhang S, Ai A, Fu Y, Kuang Y. Three-Dimensional HyCoSy With PerfluoropropaneAlbumin Microspheres as Contrast Agents and Normal Saline Injections Into the Pelvic Cavity for Morphological Assessment of the Fallopian Tube in Infertile Women. J Ultrasound Med 2017;36:741-8.

19. van Rijswijk J, van Welie N, Dreyer K, van Hooff MHA, de Bruin JP, Verhoeve HR, et al. The FOAM study: is Hysterosalpingo foam sonography (HyFoSy) a cost- 
effective alternative for hysterosalpingography (HSG) in assessing tubal patency in subfertile women? Study protocol for a randomized controlled trial. BMC Womens Health 2018;18:64.

20. Savelli L, Pollastri P, Guerrini M, Villa G, Manuzzi L, Mabrouk M, Rossi S, Seracchioli R. Tolerability, side effects, and complications of hysterosalpingocontrast sonography (HyCoSy). Fertil Steril 2009;92:1481-6.

21. Farquhar CM, Bhattacharya S, Repping S, Mastenbroek S, Kamath MS, Marjoribanks J, Boivin J. Female subfertility. Nat Rev Dis Primers 2019;5:7.

22. Luke B. Pregnancy and birth outcomes in couples with infertility with and without assisted reproductive technology: with an emphasis on US population-based studies. Am J Obstet Gynecol 2017;217:270-81.

23. Asato K, Mekaru K, Heshiki C, Sugiyama H, Kinjyo T, Masamoto H, Aoki Y. Subchorionic hematoma occurs more frequently in in vitro fertilization pregnancy. Eur J Obstet Gynecol Reprod Biol 2014;181:41-4.

24. Johnson KM, Hacker MR, Thornton K, Young BC, Modest AM. Association between in vitro fertilization and ischemic placental disease by gestational age. Fertil Steril 2020;114:579-86.

25. Modest AM, Toth TL, Johnson KM, Shainker SA. Placenta Accreta Spectrum: In Vitro Fertilization and Non-In Vitro Fertilization and Placenta Accreta Spectrum in a Massachusetts Cohort. Am J Perinatol 2020. doi: 10.1055/s-0040-1713887. [Epub ahead of print].

26. Källén B, Finnström O, Lindam A, Nilsson E, Nygren $\mathrm{KG}$, Olausson PO. Selected neonatal outcomes in dizygotic twins after IVF versus non-IVF pregnancies. BJOG 2010;117:676-82.

27. Yang M, Fan XB, Wu JN, Wang JM. Association of assisted reproductive technology and multiple pregnancies with the risks of birth defects and stillbirth: A retrospective cohort study. Sci Rep 2018;8:8296.

28. Yoshimasu K, Miyauchi N, Sato A, Yaegashi N, Nakai K, Hattori H, Arima T; Japan Environment and Children's Study Group. Assisted reproductive technologies are slightly associated with maternal lack of affection toward the newborn: The Japan Environment and Children's Study. J Obstet Gynaecol Res 2020;46:434-44.

29. Li C, Su YF, Jin L, Guo YY, Ding GL, Tang L, Cai M, Huang HF, Liu XM. Association between higher levels of serum estradiol and elevated levels of fibrin (fibrinogen) degradation products in late pregnancy following assisted reproductive technology treatment. Thromb Res 2020;187:63-71.
30. Gui J, Ling Z, Hou X, Fan Y, Xie K, Shen R. In vitro fertilization is associated with the onset and progression of preeclampsia. Placenta 2020;89:50-7.

31. Blumenfeld Z, Gleicher N, Adashi EY. Transiently increased risk of breast cancer after childbirth: implications for fertility treatments and surrogacy. Hum Reprod 2020;35:1253-5.

32. Mol BW, Tjon-Kon-Fat R, Kamphuis E, van Wely M. Unexplained infertility: Is it over-diagnosed and overtreated? Best Pract Res Clin Obstet Gynaecol 2018;53:20-9.

33. Infertility Workup for the Women's Health Specialist: ACOG Committee Opinion, Number 781. Obstet Gynecol 2019;133:e377-84.

34. Dyer S, Chambers GM, Adamson GD, Banker M, De Mouzon J, Ishihara O, Kupka M, Mansour R, ZegersHochschild F. ART utilization: an indicator of access to infertility care. Reprod Biomed Online 2020;41:6-9.

35. Cai H, Mol BW, Li P, Liu X, Watrelot A, Shi J. Tubal factor infertility with prior ectopic pregnancy: a double whammy? A retrospective cohort study of 2,892 women. Fertil Steril 2020;113:1032-8.

36. Zhu L, Zhang Y, Liu Y, Zhang R, Wu Y, Huang Y, Liu F, Li M, Sun S, Xing L, Zhu Y, Chen Y, Xu L, Zhou L, Huang H, Zhang D. Maternal and Live-birth Outcomes of Pregnancies following Assisted Reproductive Technology: A Retrospective Cohort Study. Sci Rep 2016;6:35141.

37. Henningsen AA, Gissler M, Rasmussen S, Opdahl S, Wennerholm UB, Spangsmose AL, Tiitinen A, Bergh C, Romundstad LB, Laivuori H, Forman JL, Pinborg A, Lidegaard $\varnothing$. Imprinting disorders in children born after ART: a Nordic study from the CoNARTaS group. Hum Reprod 2020;35:1178-84.

38. Hansen KR, He AL, Styer AK, Wild RA, Butts S, Engmann L, Diamond MP, Legro RS, Coutifaris C, Alvero R, Robinson RD, Casson P, Christman GM, Huang H, Santoro N, Eisenberg E, Zhang H; Eunice Kennedy Shriver National Institute of Child Health and Human Development Reproductive Medicine Network. Predictors of pregnancy and live-birth in couples with unexplained infertility after ovarian stimulation-intrauterine insemination. Fertil Steril 2016;105:1575-83.e2.

39. ElMokhallalati Y, van Eekelen R, Bhattacharya S, McLernon DJ. Treatment-independent live birth after invitro fertilisation: a retrospective cohort study of 2,133 women. Hum Reprod 2019;34:1470-8.

40. Zhang T, Li Z, Ren X, Huang B, Zhu G, Yang W, Jin L. Endometrial thickness as a predictor of the reproductive outcomes in fresh and frozen embryo transfer cycles: 
A retrospective cohort study of 1512 IVF cycles with morphologically good-quality blastocyst. Medicine (Baltimore) 2018;97:e9689.

41. Liu X, Qu P, Bai H, Shi W, Shi J. Endometrial thickness as a predictor of ectopic pregnancy in 1125 in vitro fertilization-embryo transfer cycles: a matched casecontrol study. Arch Gynecol Obstet 2019;300:1797-803.

42. Wolff EF, Vahidi N, Alford C, Richter K, Widra E. Influences on endometrial development during intrauterine insemination: clinical experience of 2,929 patients with unexplained infertility. Fertil Steril 2013;100:194-9.e1.

43. Liu WJ, Nong YQ, Ruan JX, Chen Y, Fan L, Huang QW, Liu FH. Impact of endometrial thickness during menstruation and endometrial scratching on the pregnancy in frozen-thawed embryo transfer. J Obstet Gynaecol Res 2019;45:619-25.

44. Berker B, Şükür YE, Kahraman K, Atabekoğlu CS, Sönmezer M, Özmen B, Ateş C. Impact of unilateral tubal blockage diagnosed by hysterosalpingography on the success rate of treatment with controlled ovarian stimulation and intrauterine insemination. J Obstet Gynaecol 2014;34:127-30.

45. Korsholm AS, Hvidman HW, Bentzen JG, Nyboe Andersen A, Birch Petersen K. Left-right differences in ovarian volume and antral follicle count in 1423 women of reproductive age. Gynecol Endocrinol 2017;33:320-3.

46. Fukuda M, Fukuda K, Andersen CY, Byskov AG. Rightsided ovulation favours pregnancy more than left-sided ovulation. Hum Reprod 2000;15:1921-6.

47. Lan KC, Huang FJ, Lin YC, Kung FT, Lan TH, Chang SY. Significantly superior response in the right ovary compared with the left ovary after stimulation with folliclestimulating hormone in a pituitary down-regulation regimen. Fertil Steril 2010;93:2269-73.

48. Xia W, Zhang J, Zhang D, Zhu Q, Zhang H, Huang Z, Sun F, Pan H, Duan H. Left-Right Asymmetry of Tubal Pregnancy: A 12-Year Retrospective Hospital-Based Study. J Minim Invasive Gynecol 2019;26:671-8.

49. Hou HY, Chen YQ, Chen X, Hu CX, Yang ZH, Chen

Cite this article as: $\mathrm{Gu}$ P, Yang $\mathrm{X}$, Zhao $\mathrm{X}, \mathrm{Xu} \mathrm{D}$. The value of transvaginal 4-dimensional hysterosalpingo-contrast sonography in predicting the necessity of assisted reproductive technology for women with tubal factor infertility. Quant Imaging Med Surg 2021;11(8):3698-3714. doi: 10.21037/qims-201193
J, Kong XL. Related factors associated with pelvic adhesion and its influence on fallopian tube recanalization in infertile patients. Zhonghua Fu Chan Ke Za Zhi 2012;47:823-8.

50. Canis M, Botchorishvili R, Bourdel N, Gremeau AS, Curinier S, Rabischong B. Pelvic adhesions and fertility: Where are we in 2018? J Visc Surg 2018;155 Suppl 1:S11-5.

51. Wang R, van Welie N, van Rijswijk J, Johnson NP, Norman RJ, Dreyer K, Mijatovic V, Mol BW. Effectiveness on fertility outcome of tubal flushing with different contrast media: systematic review and network meta-analysis. Ultrasound Obstet Gynecol 2019;54:172-81.

52. Van Schoubroeck D, Van den Bosch T, Meuleman C, Tomassetti C, D'Hooghe T, Timmerman D. The use of a new gel foam for the evaluation of tubal patency. Gynecol Obstet Invest 2013;75:152-6.

53. Tanaka K, Chua J, Cincotta R, Ballard EL, Duncombe G. Hysterosalpingo-foam sonography (HyFoSy): Tolerability, safety and the occurrence of pregnancy post-procedure. Aust N Z J Obstet Gynaecol 2018;58:114-8.

54. Giugliano E, Cagnazzo E, Bazzan E, Patella A, Marci R. Hysterosalpingo-contrast sonography: is possible to quantify the therapeutic effect of a diagnostic test? Clin Exp Reprod Med 2012;39:161-5.

55. Piccioni MG, Tabacco S, Merlino L, Del Negro V, Mazzeo A, Logoteta A, Del Prete F, Riganelli L, Giannini A, Monti M. Does hysterosalpingo-foam sonography have any therapeutic effect? A systematic review. Minerva Ginecol 2020;72:55-8.

56. Harrois A, Grillot N, Figueiredo S, Duranteau J. Acute kidney injury is associated with a decrease in cortical renal perfusion during septic shock. Crit Care 2018;22:161.

57. Frinking P, Segers T, Luan Y, Tranquart F. Three Decades of Ultrasound Contrast Agents: A Review of the Past, Present and Future Improvements. Ultrasound Med Biol 2020;46:892-908.

58. Exalto N, Emanuel MH. Clinical Aspects of HyFoSy as Tubal Patency Test in Subfertility Workup. Biomed Res Int 2019;2019:4827376. 